Grigg, G. W. (1958). J. gen. Microbiol. 19, 15-22

\title{
The Genetic Control of Conidiation in a Heterokaryon of Neurospora crassa
}

\author{
BY G. W. GRIGG \\ C.S.I.R.O. Animal Genetics Section, University of Sydney, Australia
}

\begin{abstract}
SUMMARY: A microconidial non-colonial heterokaryon of Neurospora crassa was produced from a macroconidial colonial and a macroconidial non-colonial homokaryon. The macroconidial colonial strain was found to have the genetic constitution microconidial $(m)$ (the $\boldsymbol{p e}^{m}$ of Barratt \& Garnjobst, 1949) suppressor of microconidial $\left(s u^{m}\right) ; c o l-1$ and the other $m^{+} s u^{m+} ; c o l-1^{+}$. The dominance relationships of $m, s u^{m}$ and col-1 with their wild type alleles and the particular nuclear ratio in which they are present determine the microconidial non-colonial phenotype of this heterokaryon, As a further consequence of the dominance relationships between col-1 and its wild-type allele, only one of the two pleiotropic effects of the mutant gene is expressed in the heterokaryon. The enormous discrepancy between the estimates of the nuclear ratio by two different plating methods is shown to be due to an almost complete suppression of one type of homokaryotic colony by the other.
\end{abstract}

Neurospora crassa produces two types of vegetative spores, macroconidia and microconidia. These differ in size, in the number of nuclei which each contains and in the time after inoculation when they first appear (Barrett \& Garnjobst, 1949). Although the environment in which they are grown can influence the time of formation of both conidial types and the size and number of nuclei of macroconidia, the differences are sufficiently great to distinguish one from the other without ambiguity. Microconidia have only one nucleus; in macroconidia the number may vary from a mean of two when the parent culture is grown on a minimal medium to six when grown on certain 'complete' media (Huebschman, 1953).

A wild-type strain produces macroconidia almost exclusively and less than $1 \%$ of microconidia. In order to have a culture produce predominantly microconidia another gene, which suppresses macroconidiation, such as colonial-1 or $f$ luffy must be present with the microconidial gene $m$ (Barratt \& Garnjobst, 1949). Col-1; $m$ and $f ; m$ strains produce copious microconidia together with a small and somewhat variable proportion $(c .1 \%)$ of macroconidia, the former strain after incubation for 7-9 days (Barratt \& Garnjobst, 1949).

In the course of plating experiments with mixtures of histidine-dependent and histidine-independent conidia, a number of spreading prototrophic colonies appeared which were unlike either of the two strains plated. The histidine-dependent strain $(K 26.9)$ had the markers albino (al-2), histidineless (K 26), was macroconidial and had a wild-type growth rate; the prototroph (P 37) was non-albino, colonial (col-1) and macroconidial. The spreading colonies proved to be microconidial and to have a growth rate on minimal medium slightly less than that of the macroconidial histidineless strain $K 26.9$ on 
histidine-supplemented medium, but very much greater than that of the macroconidial colonial strain $(P 37)$ on minimal medium. The microconidial character of the spreading, presumably heterokaryotic, colonies was unexpected and it was therefore studied.

\section{METHODS}

All conidia before being plated were washed twice by centrifugation and resuspension in $10 \mathrm{ml}$. quantities of sterile distilled water. Dilutions were made in McCartney bottles with appropriate agitation between dilutions to disperse clumps and to distribute conidia at random in the suspending medium. The media used were of the following composition: minimal medium (Westergaard

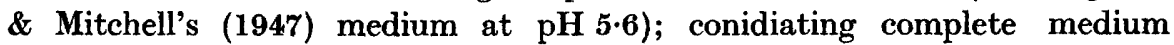
(Horowitz, 1947). Crosses were performed on Westergaard \& Mitchell's medium at $\mathrm{pH} 6 \cdot 5$.

\section{RESULTS}

The spreading colonies were proved to be heterokaryons by the following observations.

(1) Microconidia formed by the spreading colonies when plated on to appropriate media gave rise to two colony types which were identical with the two strains plated. Both types were macroconidial.

(2) Cultures with all the properties of the microconidial prototrophic spreading colonies were obtained when heterokaryons between $P 37$ and various macroconidial histidine-dependent strains were synthesized on conidiating complete medium. The histidine-dependent strains would not grow in this complete medium, despite its histidine content, presumably because of the presence of inhibitory concentrations of those amino acids which prevent histidine uptake (Haas, Mitchell, Ames \& Mitchell, 1952; Mathieson \& Catcheside, 1955).

(3) Patches of mycelium exhibiting the characteristics of one of the component homokaryons appeared, particularly on aged cultures. Heterokaryons often 'break down' in this way. The variant sector was always macroconidial and had the appearance of $P 37$ or $K 26.9$ or more frequently some mutant form of these. For example, sometimes a prototrophic albino wild growth was observed. This might have arisen by mutation at the histidineless locus in $K 26.9$ and subsequent selection in the unbalanced heterokaryon. On other occasions patches of macroconidial pink 'clumpy' mycelia characteristic of $m$; col-1+; $a l-2^{+}$(Barratt \& Garnjobst, 1949) were observed. These presumably were derived from a mutation of the col-1 in $P 37$ to $c o l-1^{+}$followed by selection for the non-colonial prototrophic nuclei. These two types of variant growth in the heterokaryon, due to unbalance brought about by mutation in one of the component strains, occurred suddenly and discretely. Another type of change resulting in increased production of macroconidia by the heterokaryon occurred gradually, presumably as a consequence of a selection for the $\boldsymbol{P} 37$ nuclei in the heterokaryon. After a number of transfers the growth habit of some stocks of the heterokaryon became progressively more colonial and 
similar to $\boldsymbol{P} 37$ and they produced fewer micro- and more macroconidia. The appearance of the heterokaryon is compared with that of the component strains $P 37$ and $K 26.9$ in Table 1.

Table 1. Comparison of the heterokaryon with its component homokaryons

\begin{tabular}{|c|c|c|c|c|}
\hline \multirow{2}{*}{$\begin{array}{l}\text { Neurospora } \\
\text { crassa } \\
\text { culture }\end{array}$} & \multicolumn{4}{|c|}{ Character } \\
\hline & $\begin{array}{l}\text { Growth } \\
\text { habit }\end{array}$ & Aerial hyphae & Conidia & Colour \\
\hline P37 & Colonial & $\begin{array}{l}\text { Short, covering whole } \\
\text { surface of colony }\end{array}$ & Macroconidia & Pink \\
\hline Heterokaryon & Spreading & None & Microconidia & $\begin{array}{l}\text { Pink-brown because } \\
\text { of masses of micro- } \\
\text { conidia covering } \\
\text { surface of culture }\end{array}$ \\
\hline K26.9 & Spreading & $\begin{array}{l}\text { Long, present mainly } \\
\text { at aerial end of tube } \\
\text { slope }\end{array}$ & Macroconidia & White \\
\hline
\end{tabular}

\section{Genotypes of the two components of the heterokaryon}

The component strains of the heterokaryon $(P 37$ and $K 26.9)$ were each examined to see whether they were heterokaryotic for $m$ or whether they contained a modifier of $m$, such that $m$; $s u^{m}$ produced macro- and not microconidia. The cross of $K 26.9$ with a colonial microconidial strain segregated 1:1 for $m$ amongst the col type ascospore isolates, indicating that $K 26.9$ did not have the gene $m$. Neither was the gene $f$ present since no fluffy colonies were observed in the 200 ascospore isolates or 250 colonies derived from single microconidia.

When P 37 was crossed with Emerson $A, m$ and another gene which has been designated $s u^{m}$, showed regular segregation. The data obtained from two crosses of $P 37$ with two wild-type strains and of $P 37$ with a microconidial strain are summarized in Table 2. Only those segregants having col-1 were

Table 2. Neurospora crassa; segregation of $s u^{m}$ in two crosses

\begin{tabular}{|c|c|c|c|c|}
\hline \multirow[b]{2}{*}{ Cross } & \multicolumn{3}{|c|}{ col-1 segregants } & \multirow[b]{2}{*}{$\begin{array}{l}\chi^{2} \text { independence } \\
\text { of } s u^{m} \text { and } m\end{array}$} \\
\hline & $\operatorname{col}-1 ; m ; s u^{m}$ & col-1; $m ;+$ & $\begin{array}{l}\text { col-1; + ; sum } \\
\text { col-1; }+;+\end{array}$ & \\
\hline \multirow[t]{2}{*}{ col-1; $m ; s u^{m} \times+;+;+$} & $\begin{array}{l}52 \\
30\end{array}$ & $\begin{array}{r}10 \\
8\end{array}$ & $\begin{array}{l}48 \\
25\end{array}$ & $\begin{array}{l}28 \cdot 5 \\
12 \cdot 7\end{array}$ \\
\hline & 82 & 18 & 73 & $41 \cdot 2$ \\
\hline \multicolumn{5}{|c|}{ Deviation $\chi^{2}=41.0, P<0.01 ;$ heterogeneity $\chi^{2}=0.2, P=0.65$} \\
\hline col-1; $m ; s u^{m} \times+; m ;+$ & 31 & 30 & - & 0.016 \\
\hline
\end{tabular}

scored since microconidia are produced only in strains having the constitution col-1; $m$. It was not possible to differentiate phenotypically between the classes $c o l-1 ; m^{+} ; s u^{m}$ and $c o l-1 ; m^{+} ; s u^{m+}$, each of which was aconidiate, and they were therefore grouped together. The data obtained from the cross of $c o l-1 ; m ; s u^{m}$ and $+; m ;+$ show equality in the col-1; $m ; s u^{m}$ and col-1; 
$m$; $s u^{m+}$ classes which would be expected if $s u^{m}$ segregated as a single gene independently of col-1, and if the classes col-1; $m ; s u^{m}$ and col-1; $m$; sut had an equal viability. The data from the coupling crosses suggest that $m$ and $s u^{m}$ are linked with a recombination percentage $p=18 \cdot 0 \pm 3.8 \%$. Because of poor fertility which has developed in the col-1; $m$; su stocks, repulsion data are not yet available.

Dominance relations of the genes $h(K 26)$, col-1, $m, s u^{m}$, and al-2

The $K 26.9$ and $P 37$ strains have the genotypes $h ; c o l-1^{+} ; m^{+} s u^{m+} ; a l-2$ and $h^{+} ; c o l-1 ; m s u^{m} ; a l-2^{+}$, respectively. The phenotypic effects of each of the mutant genes are summarized in Table 3. At the particular ratio of $K 26.9$ to $P 37$ nuclei obtaining in the heterokaryon, $h$ was 'recessive' to $h^{+}, s u^{m}$ was 'recessive' to $s u^{m+}, a l-2$ was 'recessive' to $a l-2^{+}$but $m$ was 'dominant' to $m^{+}$. The two pleiotropic effects of col-1, namely, $(a)$ the suppression of macroconidia, and the production of conidia in association with $m$, and $(b)$ the restriction of growth to a colonial habit, showed different dominance relations. In the first mutant character was 'dominant', but in the second the mutant character was 'recessive'.

Table 3. Phenotypic effects of $h$, col-1, $m, s u^{m}$ and al-2

\begin{tabular}{|c|c|}
\hline Gene & Phenotype \\
\hline $\boldsymbol{h}$ & $\begin{array}{l}\text { Requires histidine for growth. Growth in presence of histidine competitively } \\
\text { antagonized by several different amino-acids(Mathieson \& Catcheside, 1955). }\end{array}$ \\
\hline col-1 & Restricts growth habit and conidial production (see also $m$ ). \\
\hline$m$ & $\begin{array}{l}\text { In the presence of the aconidiate genes } c o l-1 \text { or fluffy }(f) \text { causes microconidia- } \\
\text { tion. In the absence of col- } 1^{+} \text {and } f^{+} \text {has morphological effect 'clumpy' } \\
\text { (Barratt \& Garnjobst, 1949). }\end{array}$ \\
\hline$s u^{m}$ & Interacts with $m$ in the presence of $c o l-1$ to cause macroconidiation. \\
\hline$a l-2$ & Albino. \\
\hline
\end{tabular}

\section{Nuclear ratio of the heterokaryon}

The nuclear ratio of Neurospora heterokaryons may be determined by plating either macro- or micro-conidia on suitable media and scoring the resulting colonies (Prout, Huebschman, Levene \& Ryan, 1953; Sansome, 1947), or by crossing the heterokaryon with another strain and determining the proportion of perithecia segregating for either one or the other of the nuclear types. In the first mentioned method the assumption is made that the nuclei are segregated into conidia at random. This assumption can only be tested experimentally when macroconidia are used (Prout, et al. 1953). A further assumption that the relative proportions of the different colony types observed reflect the relative proportions of the conidia plated can also be tested experimentally. In the second method there is the basic assumption, difficult to test experimentally, that there are no differences in fertilizing ability between component nuclei of the heterokaryon. Furthermore, this method is considerably more arduous than the conidial plating one, and was not used in our investigations. 
Since the heterokaryon being described produced uninucleate microconidia almost exclusively (only $1 \%$ macroconidia), an attempt was made to determine the nuclear ratio by plating suitable dilutions of the conidial suspension on histidine-supplemented medium (30 mg. histidine/1.). Of 6500 colonies on 200 Petri plates tested, only two were albino $\mathrm{col}_{-1} \mathrm{I}^{+}$; one was histidine dependent and the other was histidine independent. Assuming that the latter arose by back mutation from a histidine-dependent nucleus, the nuclear ratio $\left(h^{+}: h\right)$ would appear to be $1: 3250$.

An independent estimate of the nuclear ratio was obtained by determining the number of histidineless nuclei in the small proportion of macroconidia produced. Any macroconidium containing one of the rare histidineless nuclei could easily be recognized, since it would form a microconidial heterokaryon with a spreading growth habit. The total number of viable macroconidia in the mixture of conidia plated could be determined since macroconidia germinate more quickly than microconidia and form macroscopic colonies on conidiating complete medium $18 \mathrm{hr}$. before the latter. The nuclear ratio of five stocks of the heterokaryon, each of which grew from a single conidium, was determined and was found to be $0.024,0.027,0.028,0.045,0.045$, respectively. This proportion of approximately one histidineless nucleus in 30 is very different from the extimate of 1 in $\mathbf{3 2 5 0}$ from the microconidial plating experiment. The discrepancy suggested that the assumptions inherent in one of the methods were invalid. Either a high proportion of the homokaryotic $P 37$ macroconidia did not form macroscopic colonies, or most of the histidineless microconidia were suppressed on the plate. The first alternative must be rejected since the number of colonies scored approximated to the total number of macroconidia plated (obtained from a direct haemocytometer count). This leaves the other alternative that the development of histidineless colonies was prevented, presumably by the few prototrophic cells present. This possibility was tested by plating mixtures of prototrophic and histidineless conidia and observing the proportions of each type which formed viable colonies.

\section{Reconstruction experiment}

Although there was reason to believe that only one in thirty of the microconidia produced by the heterokaryon was histidineless, for technical reasons, mixtures of prototrophic non-albino and histidineless albino microconidia were mixed in the proportions of $6: 1$ and plated on medium containing $30 \mathrm{mg}$. histidine/l. (this concentration allows $K 26$ to grow at its maximum rate and was used in the heterokaryon plating experiment). The number of histidineless colonies which appeared was compared with the number on medium supplemented with a large excess of histidine $(100 \mathrm{mg}$./l.). The data are summarized in Table 4.

Double the number of histidine dependent colonies appeared on the medium containing $100 \mathrm{mg}$. histidine/l. as on the medium containing $30 \mathrm{mg}$. $/ \mathrm{l}$. The difference was highly significant, $t_{32}=6.312, P<0.01$. Thus it would seem that the presence of $h^{+}$colonies on the plate influences the growth of histidineless 
Table 4. Effect of the concentration of histidine in the medium on the suppression of growth of al-2; h conidia by $+;+$ conidia

Histidine concentration:

Colony type:

Total number of colonies: Mean number of colonies per plate:

\section{$30 \mathrm{mg} . / 1$.}

$\overbrace{\substack{a l-2 ; h \\ 119}}^{\begin{array}{c}+;+ \\ 1297\end{array}}$

$7 \cdot 934 \pm 0 \cdot 597 \quad 92 \cdot 640 \pm 2 \cdot 059$
$100 \mathrm{mg} \cdot / 1$.

\begin{tabular}{|c|c|}
\hline$a l-2 ; h$ & $+;+$ \\
\hline 280 & 1785 \\
\hline
\end{tabular}

Difference between the number of $a l-2 ; h$ colonies on medium containing 30 and $100 \mathrm{mg} / \mathrm{l}$. of histidine $=6 \cdot 804 \pm 1 \cdot 078 ; t_{32}=6 \cdot 312, P<0 \cdot 01$.

colonies present so that at levels of histidine normally sufficient for optimum growth of the histidineless strain the growth of histidineless conidia is prevented.

\section{DISCUSSION}

Forced heterokaryons may be synthesized between two auxotrophic strains which differ in two biochemical genes (Beadle \& Coonradt, 1944). Similarly, such heterokaryons may be formed between a prototrophic and an auxotrophic strain if the growth of the prototroph is restricted by the presence of a colonial gene such as col-1 and the auxotroph carries the wild-type allele of the colonial gene. The heterokaryon discussed here was of this latter type.

The colonial component $\boldsymbol{P} 37$ though macroconidial carried the microconidial gene $(m)$. It was prevented from producing exclusively microconidia by the presence of the gene $s u^{m}$ which acted in a complementary fashion with $m$ to induce macroconidiation. As a consequence of the differing dominance relations of $m$ and $s u^{m}$ and of the pleiotropic effects of col-1, the heterokaryon between two macroconidial strains $P 37$ and $K 26.9$ was microconidial and had a spreading growth habit. At ratios of $\mathrm{col}-1^{+} ; m^{+} s u^{m+}$ nuclei to $\mathrm{col}-1 ; m$ $s u^{m}$ nuclei of 0.045 and less, $m$ was dominant to $m^{+}$but $s u^{m}$ was recessive to $s u^{m+}$. The microconidial phenotype of col-1;m was dominant to $c o l-1 ; m$ but the growth habit effect of $c o l-1$ was recessive to col-1+. The differing dominance relations of $m$ compared with $s u^{m}$ and of the pleiotropic characters of the col-1 gene suggest that the genes are not equally effective in producing their phenotypic effect. Segregation of the pleiotropic effects of col-1 has not been observed in the course of a large number of crosses involving col-1 and the fact that mutation affects all the pleiotropic characters simultaneously makes it unlikely that these several characters are due to closely linked genes.

The gene $s u^{m}$ is akin to a suppressor gene since it changes the microconidial character of the strain to the wild-type macroconidial condition. Because $m s u^{m}$ does not have the same phenotype as $m^{+}$, a col-1; $m^{+}$culture being aconidiate while a col-1; $m s u^{m}$ culture is macroconidial, it is perhaps preferable to refer to it as a modifier rather than as a suppressor. It is of little use as a marker because it can be scored only when col-1 and $m$ are also present.

The validity of using the terms dominance and recessiveness in the genic sense in the heterokaryons between haploid nuclei may be queried. The terms 
were originally applied to intranuclear phenomena in diploid organisms but in heterokaryons they refer to internuclear effects. Moreover, in normal diploids, heterozygous for a particular gene, there is usually a constant $1: 1$ ratio of the two alleles, whereas in heterokaryons the nuclear ratio is usually not $1: 1$. Different heterokaryons involving the same components show considerable differences in this respect. Thus when specifying 'dominance' or 'recessiveness' in heterokaryons the nuclear ratio must also be specified. As might be expected 'dominance' varies with the nuclear ratio, thus as the proportion of col-1: $m s u^{m}$ nuclei in the heterokaryon increased the growth rate decreased and the production of macroconidia increased at the expense of the production of microconidia. This is in agreement with the observations of Barratt \& Garnjobst (1949), who found the growth rate of heterokaryons having nuclear ratios greater than $1 \mathrm{col}-1^{+}$nucleus : $1 \mathrm{col}-1$ nucleus was equal to that of the $\mathrm{col}-1^{+}$ strain but as the proportion of col-1 nuclei increased the growth rate decreased. The growth rate of the heterokaryon at a ratio of $1 \mathrm{col}-1^{+}: 100 \mathrm{col}-1$ was still 50 times that of the col-1 strain though only $0 \cdot 18$ times that of the $c o l-1^{+}$strain.

The determination by plating methods of the proportion of a particular cell type in a mixture is fraught with difficulties due to cell interactions of various sorts. Cell competition between non-growing auxotrophs and prototrophs plated on minimal medium has been described by Grigg (1952). The complex interactions between diverse types of cells on the same plate has been discussed by Harper (1950). In the present instance an attempt to determine the nuclear ratio of a microconidial heterokaryon by plating the conidia on to a histidinesupplemented medium and scoring the resulting colonies failed because the prototrophic cultures interfered in some way with the growth of the histidineless ones. Reconstruction experiments, in which growth of histidineless colonies from a mixture of prototrophic and histidineless conidia plated under similar conditions was studied, verified the existence of the suppression. For technical reasons a higher proportion of histidineless conidia was used in the reconstruction experiments than would be present in the mixed conidia from the heterokaryon. It might be expected that as the proportion of histidine-dependent conidia decreased the extent of the suppression by prototrophic conidia would increase. This would explain the almost complete suppression of the histidine-dependent conidia when in the presence of thirty times their number of prototrophs as compared with partial suppression when the proportion was 1:6. A clue to the mechanism of the suppression is given by the work of Mathieson \& Catcheside (1955) who found that prototrophs as well as histidineless cultures took up histidine from the medium against a considerable concentration gradient. If the prototrophic microconidia from the heterokaryon germinated sooner than the histidineless ones, and we have data which suggest this may be so, they might remove sufficient histidine from the low-histidine medium to prevent the normal growth of the histidine-dependent conidia. When the germination rates of conidia from the prototrophic and histidineless component strains of the heterokaryon were compared on solid medium supplemented with histidine ( 30 and $100 \mathrm{mg}$. $/ \mathrm{l}$.) the prototrophs were found to germinate significantly more rapidly than the $K 26$ conidia. 
When a large excess of histidine was present in the medium, presumably sufficient was left to enable the more slowly-germinating histidineless conidia to grow to macroscopic colony size. Other instances of colony interactions influencing the determination of nuclear ratios of heterokaryons have been observed (Jinks, 1952), and it is a factor which should be tested when conidial plating methods are employed for estimating nuclear ratios of heterokaryons, otherwise estimates of the proportion of a particular cell type in a population may be completely erroneous.

I wish to thank Mrs M. J. Mayo for her help in reading and criticising the manuscript, Professor D. G. Catcheside, F.R.S., for providing me with laboratory accommodation and critical discussion during the progress of this work and Professor Sterling Emerson for his encouragement and interest.

\section{REFERENCES}

Barratt, R. W. \& Garnjobst, L. (1949). Genetics of colonial microconidiating mutant strain of Neurospora crassa. Genetics, 34, 351.

Beadle, G. \& Coonradt, V. L. (1944). Heterocaryosis in N. crassa. Genetics, 29, 291.

GrigG, G. W. (1952). Back mutation assay method in micro-organisms. Nature, Lond. $169,98$.

Haas, F., Mitchell, M., Ames, B. N. \& Mrtchell, H. K. (1952). A series of histidineless mutants of $N$, crassa. Genetics, $37,217$.

HARPER, J. L. (1950). An investigation of the interaction of soil microorganisms with special reference to the study of the bacterial populations of plant root systems. University of Oxford, Ph.D. Thesis.

Honowitz, N. H. (1947). Methionine synthesis in Neurospora: The isolation of cystathionine. J. biol. Chem. 171, 255.

Huebschman, C. (1953). A method for varying the average number of nuclei in the conidia of Neurospora crassa. Mycologia, 44, 599.

Jinks, J. L. (1952). Heterokaryosis: a system of adaptation in wild fungi. Proc. Roy. Soc. B, 140, 83.

Mathieson, M. J. \& Catcheside, D. G. (1955). Inhibition of histidine uptake in Neurospora crassa. J. gen. Microbiol. 13, 72.

Prout, T., Huebschman, C., Levene, H. \& Ryan, F. J. (1953). The proportions of nuclear types in Neurospora heterokaryons as determined by plating conidia. Genetics, 38, 518.

SANSOME, E. R. (1947). Somatic segregation by microconidial isolation in synthesised heterokaryons of Neurospora crassa. Proc. roy. Soc. Edinb. B, 62, 299.

WestergaArd, M. \& Mrtchell, H. K. (1947). Neurospora V. A synthetic medium favouring sexual reproduction Amer. J. Bot., 34, 573. 